\title{
Part Assembly Using Static and Dynamic Force Fields
}

\author{
Jiangchun Luo \\ Lydia Kavraki \\ Department of Computer Science, Rice University \\ 6100 Main Street, Houston, TX 77005 \\ luojc|kavraki@cs.rice.edu
}

\begin{abstract}
Part assembly is an important goal of part manipulation. Among other techniques, programmable force fields have been introduced for part manipulation. Many different force fields have been proposed to manipulate a part, such as the squeeze and the elliptical fields. For part assembly, more than one part needs to be manipulated. This can create problems since there can be interactions between the parts such as impact and friction. With technology developing, certain fields can be implemented in the micro-scale with MEMS actuator arrays and in the macro-scale with arrays of directed air jets or small motors. Modern technology is beginning to provide the means to control the magnitude and frequency of each actuator of the implemented force field. Thus dynamic and localized force fields can be used for part manipulation. This paper presents a novel strategy to assemble two parts with a sequence of static and dynamic programmable force fields. The strategy involves some initial sensing. Uncertainties occurring in the motion of the parts are taken into account to make the proposed strategy more robust.
\end{abstract}

\section{Introduction and Related Work}

Part assembly is an important but time-consuming operation in industrial automation. To assemble two parts, first we need to understand how to manipulate each part individually. Several techniques and devices have been proposed for part manipulation, some of them involving sensing [1] and others requiring no sensing [6]. We concentrate on methods that use programmable force fields and propose a novel strategy to assemble two parts, which requires an initial sensing operation.

\subsection{Problem Description}

Consider two laminar parts on a plane. The problem is to find a sequence of force fields to assemble them. There are three basic assumptions in our work:

- Assumption 1: one part lies in the left half plane and the other lies in the right half plane, as shown in Figure 1(a).

- Assumption 2: we know the initial orientations of the two parts but do not know their initial positions.

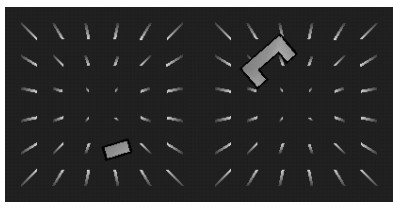

(a)

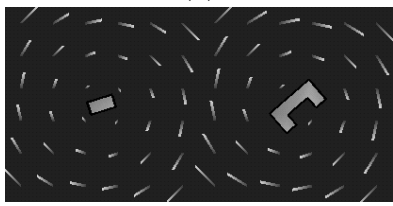

(c)

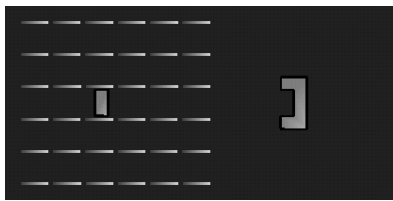

(e)

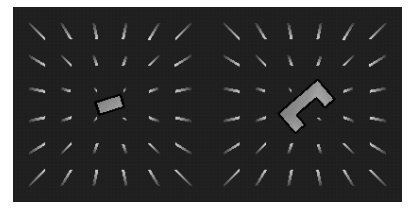

(b)

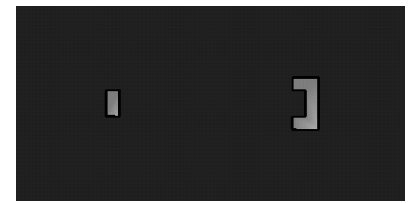

(d)

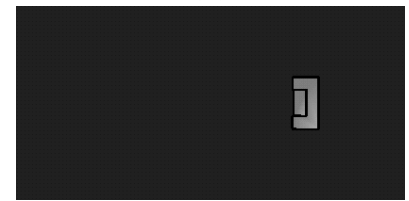

(f)
Figure 1: Snapshots along a part assembly simulation, (a) shows the initial positions and orientations of two parts before assembly, (b) shows the parts after centering with radial fields, (c) shows curl force fields applying to the parts, (d) shows the parts with the assembly orientations after rotation, (e) shows a push force field applied to one part, and (f) shows the assembled two parts.

- Assumption 3: the assembly positions and orientations of the two parts are known.

This paper focuses on the case where one part has a convex section and the other part has a complementary concave section. We call the part with the convex section "inserting part" $P_{1}$ and the other part "inserted part" $P_{2}$. If both of the parts are convex, for example two rectangles, it makes no difference which part is called the inserting part.

\subsection{Related Work}

Extensive research has been done for manipulating a single part. The use of programmable force fields is a new but rapidly developing solution. Force fields can be used for a wide variety of parts, require little or no sensing, employ simple devices and are rather robust $[4,10]$. The basic idea is following: the field is realized on a planar surface on which the part is placed. The force and torque applied on the part translate and rotate it. Donald and Böhringer pio- 
neered the algorithmic study of programmable force fields for part manipulation. In recent papers many kinds of force fields have been proposed. One interesting class of force fields are potential fields in which the work integral is zero along any circular path. Such fields are the squeeze field $[3,4,11]$, the elliptical field $[3,7,11]$, and the radial plus gravity field $[3,8]$. These potential fields can translate and rotate a part to equilibrium configurations. All current force fields only handle a single part. When placing two parts in the same force field, the problem becomes much more complicated because there could be impact and friction between the parts. For the purposes of this paper, we put one part in each half plane and apply force fields on each half plane individually to avoid the interactions between the parts.

From the technology point of view, certain force fields can be implemented in the micro-scale with MEMS actuator arrays [2] and in the macro-scale with arrays of directed air jets [15], MDMS [11] and vibrating plates [14]. In particular, the magnitude and frequency of each air jet can be controlled individually. This technology inspires us to think about using dynamic and localized force fields. The dynamic force fields are time-varying; i.e., the magnitude and direction of the force at each point can change with time. The squeeze, elliptical, and radial plus gravity force fields are all static, not dynamic.

\subsection{Our Approach}

To avoid part interactions, we divide the assembly procedure into three stages. It proceeds as follows:

(1) Center the two parts in their half planes with separate radial fields; see Figure 1 (a) and (b).

(2) Rotate the parts to their assembly orientations with two dynamic curl fields; see Figure 1 (c) and (d).

(3) Translate the inserting part to complete the assembly with 1 or 2 dynamic push fields; see Figure 1 (e) and (f).

We use sensing at the very beginning to find the initial orientations of the two parts. After that, the whole process does not require any sensing. However, sensing, if available, can be used to compensate for errors.

Rotation and translation control are critical in the above strategy. The parts may start with any initial orientation, so the rotation force field has to be able to rotate each part to a specified final orientation. Currently, there are three proposed potential fields which can be used to orient parts. They are the squeeze, elliptical, and radial plus gravity fields. A polygonal part can be uniquely oriented up to symmetries with a sequence of squeeze fields [3, 4]. However, we have to select different sequences of squeeze fields for different parts. Furthermore, the parts can only be oriented up to symmetries of the convex hull of the part. The elliptical field can orient "asymmetric" parts to two stable orientations [3, 7]. But still the final orientations are not unique. Also, for some symmetric parts such as squares, the elliptical force field can not orient them. The radial plus gravity force field can orient a part whose pivot point is different than its center of mass to an unique orientation $[3,8]$. But there are still several problems. First, so far there is no rule about how to select the magnitude of the gravity field. Second, to calculate a pivot point of a part under a radial field is not an easy job. Last, parts with the same pivot point and center of mass may have more than one final orientation. Since current force fields can not control the rotation of the part easily, this paper presents a dynamic curl field to reach this goal. We also present a pure radial field and a dynamic push field to control part translation. We will show that the two force fields can translate a part without rotating it.

\section{Notation and Related Background}

\subsection{Notation}

Consider a part $\mathrm{P}$ moved under a force field $\mathrm{W}$. The center of mass of $\mathrm{P}$ is called COM for simplicity. We are going to use the following symbols:

- $\rho, M, S, I_{z}$ are the mass density, mass, area, and moment of inertia of $\mathrm{P}$;

- $x o y, X O Y, \mathbf{r}$ are the global coordinate system, the local coordinate system, and $\mathbf{r}=(X, Y)^{T}$;

- $\left(x_{c}, y_{c}\right)^{T}, \mathbf{v}_{c}, \alpha_{c}$ are the global coordinates, translational velocity and acceleration of the COM of P;

- $\mathbf{f}(x, y), \mathbf{F}, T$ are the force at a point (x, y), the external force and external torque applied on $\mathrm{P}$ by $\mathrm{W}$.

$-\mathbf{f}_{d}, \mathbf{F}_{d}, \mathbf{T}_{d}$ are the damping force at a point, the damping force and torque applied on P.

- $\mathbf{v}, a, \omega, \alpha$ are translational velocity, translational acceleration, angular velocity, and angular acceleration of $\mathrm{P}$.

\subsection{Basic Dynamics for Planar Motion}

The motion of $\mathrm{P}$ under $\mathrm{W}$ is a standard planar motion. Any planar motion of $\mathrm{P}$ can be decomposed into a pure translation of its COM and a pure rotation about its COM. For the translation, using Newton's second law, we get

$$
\mathbf{F}+\mathbf{F}_{d}=M \mathbf{a} .
$$

For the rotation, there is a similar equation in dynamics:

$$
\mathbf{T}+\mathbf{T}_{d}=I_{z} \alpha .
$$

Using the two fundamental dynamics equations, we can develop the differential equations for translation and rotation by substituting specific $\mathbf{F}, \mathbf{F}_{\mathrm{d}}, \mathbf{T}$, and $\mathbf{T}_{\mathrm{d}}$.

\subsection{Damping Force and Torque}

There are very few papers that discuss the damping model of programmable force fields. However, without damping, no potential field can orient and position a part. Since the potential energy is a conservative energy, without 


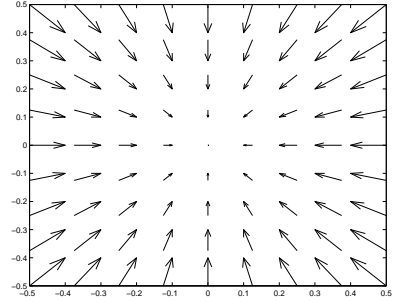

(a)

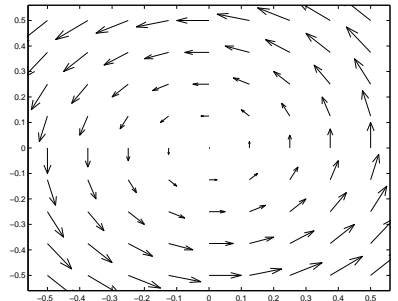

(b)
Figure 2: (a) is a radial force field with $\beta=1$. (b) is a curl force field with $\beta=1$.

damping, the sum of a part's potential and kinematic energy will never decrease, which means the part will never stop if its initial energy is not minimum. Damping plays a critical role in our part assembly method. There are two kinds of damping in macro-scale physical systems (1) Coulomb or dry friction damping and (2) Viscous or fluid damping [13]. With Coulomb friction, the magnitude of maximum static friction force applied on a part $\mathrm{P}$ is of the form $F_{d m}=\mu N$, where $\mu$ is the Coulomb friction coefficient and $\mathrm{N}$ is the normal force, which is the gravity of $\mathrm{P}$ in this case. Because both $\mu$ and $\mathrm{N}$ are constants, $F_{d m}$ of $\mathrm{P}$ is a constant and hence is a threshold value for translating P. Any external force less than $F_{d m}$ can not translate P. For the same reason, the Coulomb friction torque is also a threshold value for rotating P. So Coulomb friction is not a suitable model for force fields. In this paper we use viscous damping. At each point of the part, the damping force is

$$
\mathbf{f}_{d}=-k \cdot \mathbf{v},
$$

where $k$ is the damping coefficient, which is a constant under the same force field. Luntz and Messner use a similar friction model [11]. The damping force and torque over the whole part are

$$
\begin{gathered}
\mathbf{F}_{d}=\int_{S}-k \mathbf{v} d s=-k S \mathbf{v}_{c}, \\
\mathbf{T}_{d}=\int_{S}-k \mathbf{r} \times \mathbf{v} d s=-\frac{k \omega}{\rho} I_{z} \cdot \vec{k} .
\end{gathered}
$$

\section{Part Assembly with Force Fields}

As discussed in the introduction, the rotation and translation control of the parts are key to assemble two parts. We present a dynamic curl force field to control parts' rotation and a dynamic push force field to control the inserting part's translation. Before that, some preprocessing is needed to center the two parts in their own half planes. We present a radial force field for centering the parts.

\subsection{Centering Parts Using Radial Fields}

A radial force field is of the form:

$$
f(x, y)=(-\beta x,-\beta y),
$$

where $\beta$ is a constant. Our strategy use radial force fields with $\beta=1$. Figure 2 (a) shows such a radial field.

Part Translation under Radial Fields It is known that the radial field is a potential field $[3,8]$. The force applied on a part is

$$
\left(F_{x}, F_{y}\right)^{T}=\left(-S x_{c},-S y_{c}\right)^{T} .
$$

Any part has a stable translational equilibrium position, at which $F_{x}=0$ and $F_{y}=0$. Thus the equilibrium condition is equivalent to the COM of the part being equal to zero. In $\mathrm{x}$ direction, plugging $F_{x}, F_{d x}$ (damping force in $\mathrm{x}$ direction), $v_{x c}=\dot{x_{c}}$, and $a_{x c}=\ddot{x_{c}}$ into Equation 1, we get

$$
\rho \ddot{x_{c}}+k \dot{x_{c}}+x_{c}=0 .
$$

Suppose the initial position of $\mathrm{P}$ is $\left(x_{0}, y_{0}\right)^{T}$ and the initial velocity of $\mathrm{P}$ is zero. We solve Equation 8 with the initial conditions $\dot{x_{c}}(0)=0$ and $x_{c}(0)=x_{0}$ :

$$
x_{c}(t)=e^{-\frac{k t}{2 \rho}}\left[x_{0} \cos (\lambda t)+\frac{k x_{0}}{4 \rho-k^{2}} \sin (\lambda t)\right],
$$

where $\lambda=\frac{\sqrt{4 \rho-k^{2}}}{2 \rho} \cdot y_{c}(t)$ is almost the same as $x_{c}(t)$, except for $\frac{y_{c}(t)}{x_{c}(t)}=\frac{y_{0}}{x_{0}}$. This proves that the trajectory of the COM of the part is a straight line and, of course, it will oscillate about the origin of the force field a few times. Also from Equation 9, if the two parts have the same density $\rho$, they will have the same oscillation frequency, which guarantees that they will reach the origins of their half planes at the same time.

Lack of Rotation under Radial Fields From Equation 2 , if no torque is applied on the part, the part can not be rotated. The torque about the COM of the part is $\mathbf{T}=$ $\int_{S} \mathbf{r} \times \mathbf{f}(r) d s$. Under the XOY coordinate system centered at the COM of the part, $\mathbf{r}=X \cdot \vec{i}+Y \cdot \vec{j}$. Using coordinate translation, we get

$$
\mathbf{T}=x_{c} S Y_{c}-y_{c} S X_{c}=0 .
$$

The reason why $\mathbf{T}=0$ is $\left(X_{c}, Y_{c}\right)^{T}=(0,0)^{T}$. Thus the radial field does not apply a torque on the part. If the part has zero initial angular velocity, it is trivial to prove that the damping torque is also zero because of zero external torque.

\subsection{Rotating Parts Using Dynamic Curl Fields}

Since each part has an arbitrary initial orientation, we need a force field to rotate the part to any desired orientation. To reach this goal, a curl around the COM of the part can be used; see Figure 2 (b). Consider a dynamic force field of the form:

$$
\mathbf{f}(x, y)=(1-\sin t)(-\beta y, \beta x)^{T}, t \in\left[0, \frac{\pi}{2}\right] .
$$

One consideration regarding the dynamic term is that, at $t=\frac{\pi}{2}, f(x, y)=(0,0)^{T}$ and $f^{\prime}(x, y)=(0,0)^{T}$ in order for the curl field to fade smoothly. Also, the trigonometric wave is easy to generate in real implementations. 
Lack of Translation under Curl Fields Suppose the $\mathrm{COM}$ of the part is located at the origin of the curl field; i.e., $\left(x_{c}, y_{c}\right)^{T}=(0,0)^{T}$. That is why we center the parts first. We position them approximately so that a curl field can be used afterwards. The $\mathrm{x}$-force applied on the part is

$$
F_{x}=-\int_{S}(1-\sin t) \beta y d x d y=-(1-\sin t) \beta y_{c} S=0 .
$$

For the same reason, $F_{y}=0$. So the external force applied on the part is zero. Furthermore, from Equation 5, if the initial velocity of the COM is $0, \mathbf{F}_{d}=0$ at all times. Thus the part will not be translated by the curl field.

Rotation Control Using Curl Fields To calculate the rotated angle, we proceed in 2 steps as follows:

- Step 1: Both the external torque (from the curl field) and the damping torque apply on the part, for $t \in\left[0, \frac{\pi}{2}\right]$. The external torque is $\mathbf{T}(t)=\int_{S} \mathbf{r} \times \mathbf{f} d s=(1-\sin t) \frac{\beta}{\rho} I_{z} \cdot \vec{k}$. Plugging $\mathbf{T}(\mathrm{t})$ and $\mathbf{T}_{\mathrm{d}}(\mathrm{t})$ in Equation 2, we get

$$
\dot{\omega}_{1}(t)+\frac{k}{\rho} \omega_{1}(t)=(1-\sin t) \frac{\beta}{\rho} .
$$

Solve Equation 12 with the initial condition $\omega(0)=0$ :

$$
\omega_{1}(t)=C_{1} \cos t+C_{2} \sin t+C_{3}-\left(C_{1}+C_{3}\right) e^{-\frac{k}{\rho} t},
$$

where $C_{1}=\frac{\beta \rho}{\rho^{2}+k^{2}}, C_{2}=-\frac{\beta k}{\rho^{2}+k^{2}}, C_{3}=\frac{\beta}{k}$. The rotated angle during step 1 is $\theta_{1}=\int_{0}^{\frac{\pi}{2}} \omega_{1}(t) d t$.

- Step 2: Only the damping torque is in effect, for $t \in$ $\left(\frac{\pi}{2},+\infty\right)$. The differential equation for $\omega_{2}(t)$ is

$$
\dot{\omega}_{2}\left(t-\frac{\pi}{2}\right)=-\frac{k}{\rho} \omega_{2}\left(t-\frac{\pi}{2}\right), t \in\left(\frac{\pi}{2},+\infty\right) .
$$

We solve it with the initial condition $\omega_{2}\left(\frac{\pi}{2}\right)=\omega_{1}\left(\frac{\pi}{2}\right)$ and get $\omega_{2}(t)=\omega_{1}\left(\frac{\pi}{2}\right) e^{-\frac{k}{\rho} t}$. The rotated angle during step 2 is $\theta_{2}=\int_{\frac{\pi}{2}}^{\infty} \omega_{2}(t) d t$

So, the total rotated angle is

$$
\theta=\theta_{1}+\theta_{2}=\beta \frac{\pi-2}{2 k} .
$$

Let us define the damping reciprocal $\eta=\frac{\pi-2}{2 k}$. Since $k$ is a constant under the same force field, $\eta$ is a constant. Thus we can easily control the rotated angle by properly selecting the value of $\beta$, which is the magnitude of the curl field. For example, if $k=0.3$ and a part need to be rotated by $\theta=\pi$, $\beta=\frac{\theta}{\eta}=\frac{0.6 \pi}{\pi-2} \approx 1.65$.

\subsection{Assembling Parts Using Dynamic Push Fields}

The last step is to translate the inserting part $P_{1}$ from its current position to its assembly position. $P_{1}$ stays at the origin of its own half plane after rotation. It is desired to only translate $P_{1}$ but not to move the inserted part $P_{2}$.
Before deciding which translation field to use, let's point out that a straight-line trajectory of $P_{1}$ is easier to control than a curved-line trajectory. Another consideration is the geometric information of the two parts. If the assembly position of $P_{1}$ is on the global x-axis, we call the pair of assembled parts a "symmetric" pair (see Figure 3 (a)) and a single translation can complete the assembly; otherwise, we call them an "asymmetric" pair (see Figure 3 (b)) and two translations are needed.

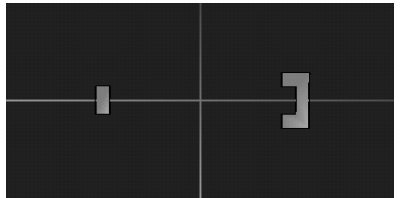

(a)

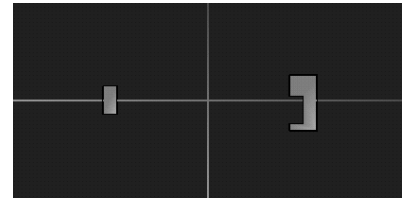

(b)
Figure 3: (a) is a symmetric pair of parts. (b) is an asymmetric pair of parts.

From the previous section, we know that the radial field can translate parts. If a global radial field has the same origin as the curl field that is used to rotate $P_{2}$ does, the radial field can only translate $P_{1}$ without moving $P_{2}$. However, this can only assemble symmetric pairs of parts, not asymmetric pairs. Under the radial field, a part is always translated toward the origin of the field. In this case, the trajectory of $P_{1}$ is on the global x-axis since its current position is on global $\mathrm{x}$-axis; see Figure 3. For an asymmetric pair of parts, the assembly position of $P_{1}$ is not on the global $\mathrm{x}$-axis(see Figure 3 (b)) and the assembly can not be completed successfully. We propose a dynamic push force field to do the translation control, which is only in effect in the half plane of $P_{1}$. A dynamical push force field is of the form:

$$
\mathbf{f}=\gamma(1-\sin t) \cdot \mathbf{n},
$$

where $\gamma$ is the magnitude of the field and $\mathbf{n}$ is unit vector on the direction of translation, with the form of $\mathbf{n}=\cos \phi \cdot \vec{i}+$ $\sin \phi \cdot \vec{j} . \phi$ is the angle between $\mathbf{n}$ and $\mathrm{x}$-axis.

Translation Control Using Push Fields First, this field can not rotate a part, since the external torque is

$\mathbf{T}(t)=\int_{S} \mathbf{r} \times \mathbf{f} d s=\gamma(1-\sin t)\left[\sin \phi X_{c} S-\cos \phi Y_{c} S\right] \cdot \vec{k}=0$.

Furthermore, because the angular velocity is zero after rotation, the damping torque is always zero, the same reason why $\mathbf{F}_{d}=0$ under the curl field.

The translation control is almost the same as the rotation control. It proceeds as the following two steps.

- Step 1: Both the external force and the damping force apply on the part, for $t \in\left[0, \frac{\pi}{2}\right]$; the translated distance is $d_{1}=\int_{0}^{\frac{\pi}{2}} v_{c 1}(t) d t$.

- Step 2: Only the damping force is in effect, for $t \in$ $\left(\frac{\pi}{2},+\infty\right)$; the translated distance is $d_{2}=\int_{\frac{\pi}{2}}^{\infty} v_{c 2}(t) d t$. 
So, the total translated distance is

$$
d=d_{1}+d_{2}=\gamma \frac{\pi-2}{2 k}=\gamma \eta
$$

where $\eta$ is the same damping reciprocal as the one in the curl field. There are two important points for translation control. First, to complete the assembly, in general, two push fields are needed; the first one is along y direction and the second one is along $\mathrm{x}$ direction. Second, the push fields are in effect only on $P_{1}$ 's half plane, which means $P_{2}$ will not be moved. For the vertical translation, this is not an issue since $P_{1}$ is not going outside its half plane. During the horizontal push, since the push field applies on $P_{1}$ only in $d_{1}$, the field does not need to exist in $d_{2}$ during the whole process. So if $\frac{d_{2}}{d}>\frac{1}{2}$, the field in $P_{1}$ 's half plane will guarantee the correct results. Generally this is true(mathematical proof is omitted here).

\section{Motion Uncertainty}

\subsection{Involved Uncertainties}

In previous sections, we assumed that the force fields and the motions of the parts can be perfectly controlled. However, for any real implementation, uncertainty should be taken into account. A lot of research has addressed the uncertainty issue $[5,9]$. For part assembly, even though we only use sensing to find the initial orientations of the assembled parts, we still need to consider the following uncertainties mainly because of the force field control.

- Position uncertainty, see Figure 4 (a);

- Orientation uncertainty, see Figure 4 (b);

- Velocity uncertainty, see Figure 4 (c).

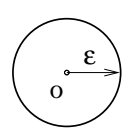

(a)

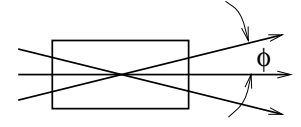

(b)

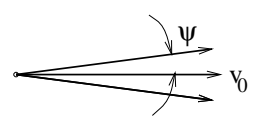

(c)
Figure 4: Three kinds of motion uncertainties. (a) shows position uncertainty. (b) shows the orientation uncertainty. (c) shows the velocity uncertainty.

We consider all three motion uncertainties during the horizontal push of $P_{1}$ in the last stage because these uncertainties directly decide whether the assembly will succeed or not. However, the uncertainties exist in all three stages of part assembly and the uncertainties in the previous stages affect those in the later stages. So all three uncertainties in the final horizontal push accumulate from centering, rotation, possible vertical translation and the horizontal push itself. Furthermore, we only need to take into account the uncertainties of $P_{1}$ because the uncertainties of $P_{2}$ can be transformed to relative uncertainties of $P_{1}$ with respect to $P_{2}$. The problem becomes similar with a classical uncertainty problem, namely the peg-in-hole problem $[5,9]$.

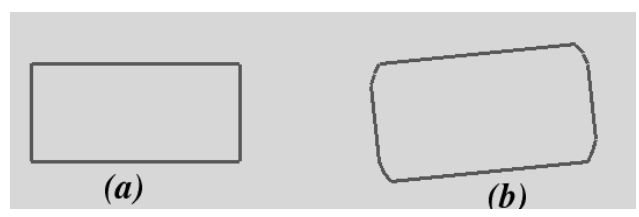

Figure 5: (a) is a rectangular part. (b) shows a box to contain the part with all possible orientations when the uncertainty $\phi=0.1$.

In order to assemble two parts, there should exist a goal region of $P_{1}$ and this goal region has to be larger than $P_{1}$. If the goal region is much larger than $P_{1}$, we can call this kind of assembly gross assembly; otherwise, we call it precise assembly. To describe the size difference, let's define the size difference rate $\delta=\frac{S_{g}-S_{p}}{S_{p}}$, where $S_{g}$ is the size of the goal region and $S_{p}$ is the size of $P_{1}$. A threshold value of $\delta$, in $(0,1)$, is helpful to distinguish precise assembly and gross assembly; say 0.2 .

\subsection{Algorithm Handling Uncertainties}

We present an algorithm as follows to resolve the horizontal push of $P_{1}$ when considering uncertainties.

- Consider $P_{1}$ as a robot and $P_{2}$ as an obstacle. Calculate the configuration space of the robot [9].

- Calculate the goal kernel, in the configuration space, from the goal region of the robot by considering the position uncertainty of the robot.

- Calculate the preimage of the goal kernel using Erdmann's backprojection algorithm [5].

- If the position uncertainty disk of the robot before the last push operation is contained inside the preimage, the assembly is guaranteed to succeed.

Many implementation details have to be omitted because of the size limit of this paper. For the C-obstacle calculation, the $\mathrm{COM}$ of the robot is selected as the reference point. Before calculating the C-obstacle, we need to enlarge the robot because of the orientation uncertainty. One solution is to calculate the minimal box to contain the robots with two extreme orientations, as shown in Figure 5. Generally, the obstacle is not convex and needs to be decomposed into a set of convex polygons first. For the goal kernel calculation and Erdmann's algorithm, please refer to the related work $[5,9,12]$. Figure 6 gives a rough idea how the preimage, goal kernel and C-obstacle look in a gross assembly.

\section{Conclusion and Future Work}

Our strategy provides a feasible solution for part assembly. During the whole analysis of the force fields, we have not made any assumptions about the shape of the parts; i.e., any pair of parts can be assembled with the strategy. We have built a simulator to illustrate several assembly operations. All three kinds of force fields used in our approach are simple and hence we expect that practical implementations will be possible. For the radial field, both MEMS and 


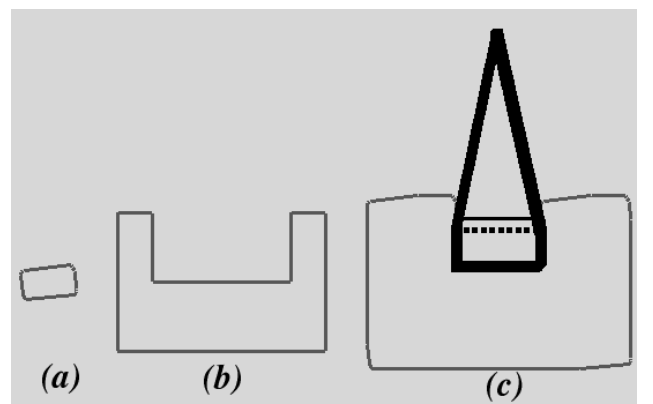

Figure 6: (a) is the same box as shown in Figure 5 (b). (b) is the inserted part(obstacle in this case). (c) shows the Cobstacle(the light line), the goal region(the area below the horizontal black solid line), the kernel of the goal region(the area below the dotted line) and the preimage of the goal kernel(the thick line).

arrays of the air jets can be used. For the dynamic curl and push fields, the air jet arrays may be more appropriate since they can control the magnitude and frequency of each air jet. Furthermore, the manipulation at each stage is simple, as shown in Table 1.

\begin{tabular}{|c|c|c|c|c|}
\hline task & field & dynamic & plan steps & parameter \\
\hline centering & radial & no & 1 & none \\
\hline rotation & curl & yes & 1 & $\beta$ \\
\hline translation & push & yes & 2 at most & $\gamma$ \\
\hline
\end{tabular}

Table 1: Fields for assembly using force fields

In the future we would like to investigate the following open questions:

- Does there exist a strategy with fewer stages which can assemble two parts with force fields? The assembly characteristics are critical here, since the two parts have to be with their assembly orientations and $P_{1}$ has to align to its assembly position before the last horizontal push.

- How can we model the interaction between the two parts when placing them inside the same field? Impact and friction between the parts are two main interactions.

- Can we find another more realistic damping model? It seems to us that Coulomb friction is more realistic. However, we have proved that Coulomb friction is not suited for the potential fields and the experimental observation shows that the parts indeed reach their desired configurations [12]. - What if the two parts are in the same half plane at the beginning? A part separation preprocessing is needed to move the two parts to different half planes. In general, part separation is an important problem itself, which could be as hard as part assembly.

\section{Acknowledgments}

The authors would like to thank Karl Böhringer, Bruce Donald, Jean-Claude Latombe, Florent Lamiraux and Attawith Sudsang for their discussions. Work on this paper by Lydia Kavraki and Jiangchun Luo is partially supported by NSF IRI-970228, NSF CISE SA1728-21122N and a Sloan Fellowship to Lydia Kavraki.

\section{References}

[1] S. Akella and M. T. Mason. Parts orienting with partial sensor information. In Proc. IEEE Int. Conf. on Robotics and Automation (ICRA), 1998.

[2] K.-F. Böhringer and B. R. Donald. Algorithmic MEMS. In P. K. Agarwal, L. Kavraki, and M. Mason, editors, Robotics: The Algorithmic Perspective. A. K. Peters, Ltd, Wellesley, MA, 1998. Accepted for publication. Forthcoming.

[3] K.-F. Böhringer, B. R. Donald, L. Kavraki, and F. Lamiraux. Part orientation with one or two stable equilibria using programmable vector fields. IEEE Transactions on Robotics and Automation, 1999. Submitted for review.

[4] K.-F. Böhringer, B. R. Donald, and N. C. MacDonald. Upper and lower bounds for programmable vector fields with applications to MEMS and vibratory plate parts feeders. In International Workshop on Algorithmic Foundations of Robotics (WAFR), Toulouse, France, July 1996.

[5] M. A. Erdmann. On motion planning with uncertainty. Master's thesis, Massachusetts Institute of Technology, Cambridge, MA, 1984.

[6] K. Y. Goldberg. Orienting polygonal parts without sensing. Algorithmica, 10(2/3/4):201-225, August/September/October 1993.

[7] L. Kavraki. Part orientation with programmable vector fields: Two stable equilibria for most parts. In Proc. IEEE Int. Conf. on Robotics and Automation (ICRA), Albuquerque, New Mexico, Apr. 1997.

[8] L. Kavraki and F. Lamiraux. Positioning symmetric and non-symmetric parts using radial and constant force fields. In International Workshop on Algorithmic Foundations of Robotics (WAFR), 2000.

[9] J.-C. Latombe. Robot Motion Planning. Kluwer Academic Publishers, Boston, MA, 1991.

[10] W. Liu and P. Will. Parts manipulation on an intelligent motion surface. In IEEE/RSJ Int. Workshop on Intelligent Robots \& Systems (IROS), Pittsburgh, PA, 1995.

[11] J. E. Luntz, W. Messner, and H. Choset. Discrete actuator array vectorfield design for distributed manipulation. In Proc. IEEE Int. Conf. on Robotics and Automation (ICRA), Detroit, Michigan, May 1999.

[12] J. Luo. Part assembly using static and dynamic force fields. Master's thesis, Rice University, Houston, Texas, June, 2000.

[13] B. B. Muvdi, A. W. Al-khafaji, and J. W. McNabb. Dynamics for Engineers. Springer-Verlag New York, New York, USA, 1997.

[14] D. Reznik and J. F. Canny. Universal part manipulation in the plane with a single horizontally vibrating plate. In P. K. Agarwal, L. Kavraki, and M. Mason, editors, Robotics: The Algorithmic Perspective. A. K. Peters, Ltd, Wellesley, MA, 1998.

[15] M. Yim and A. A. Berlin. Contact and non-contact mechanisms for distributed manipulation. In ICRA Workshop at Distributed Manipulation, May 1999. 